\title{
LINC01121 Inhibits Cell Apoptosis While Facilitating Proliferation, Migration, and Invasion Though Negative Regulation of the Camp/PKA Signaling Pathway via GLP1R
}

Yi-Gang Qian ${ }^{a, b, c}$ Zhou Ye ${ }^{a, b, c}$ Hai-Yong Chen ${ }^{a, b, c}$ Zhen Lv ${ }^{a, b, c}$ Ai-Bin Zhang ${ }^{a, b, c}$ Le Fann ${ }^{a, b, c}$ Jie Zhou ${ }^{a, b, c}$ Shu-Sen Zheng ${ }^{a, b, c}$ Wei-lin Wang ${ }^{d}$

aDivision of Hepatobiliary and Pancreatic Surgery, Department of Surgery, First Affiliated Hospital, School of Medicine, Zhejiang University, Hangzhou, 'Key Laboratory of Combined Multi-organ Transplantation, Ministry of Public Health, Key Laboratory of Organ Transplantation, Zhejiang Province, Hangzhou, 'Collaborative Innovation Center for Diagnosis and Treatment of Infectious Diseases, Hangzhou, 'Key Laboratory of Precision Diagnosis and Treatment for Hepatobiliary and Pancreatic Tumor of Zhejiang Province, First Affiliated Hospital, Zhejiang University School of Medicine, Hangzhou, China 